\title{
Research and Systematic Design on Autonomous Mission Management Technology of Agile Satellite
}

\author{
Zhu Jian-bing, Zhao Wei, Wang Lu-yuan and LI Wen-juan \\ CAST, Beijing Institute of Spacecraft System Engineering, 100094, Youyi Road HaiDian District No.104, China
}

\begin{abstract}
Compared to traditional non-agile imaging satellites, the imaging efficiency of agile satellites are far higher and the imaging requirements are correspondingly more sophisticated, which in turn calls for better design and implementation of the onboard autonomous mission management system. According to the characteristics of the autonomous mission management system of agile satellites, this paper presents and illustrates the functional modules and key technology required by the autonomous mission management system. This paper proposes a layered architecture design, which employs a progressive and gradually detailing way to facilitate the systematic modular design and to effectively reduce the complexity of the implementation of the design. Combined with mission plan, emergency replan, image parameter real-time calculation technique, autonomous monitoring technique of plan implementation and other techniques, this design makes the agile imaging mission more scientifically designed, properly scheduling and safely implemented.
\end{abstract}

\section{Introduction}

With the advancement of space technology, the operating mode of remote sensing satellites has changed from traditional passive scanning of single degree of freedom to active scanning which change directions freely in roll, pitch and yaw axis. This considerably enhances the imaging efficiency and imaging quantity. This new type of imaging satellites is internationally called agile satellite, the typical examples of which are the American WorldView, the French Pleiades, the Britain TopSat, etc. The traditional satellite control and management mode of "ground planning and onboard implementation" can't fulfill the need of multi-mode and multi-mission imaging per revolution. Therefore, all the space powers are carrying out research on autonomous mission management technology. Based on EO-1 satellites, NASA has developed two mission plan system of satellites, ASPEN [1] and CASPER [2]. ASPEN can generate daily command of EO-1 automatically by users' mission. CASPER, by using a real-time replanning mechanism, achieves the goal of recovering the real-time condition of the system and new objections on a fixed time step or event step and realizes necessary replans based on the this real-time information. PROBA [3], supported by ESA, studied and validated certain space-borne autonomous planning techniques. Trials on autonomous mission plan system of satellites [4] are also done by FSA. Many domestic scholars and engineers as well focuses on the research on remote sensing satellite mission plan technology. HE Ren-jie and LI Ju-fang from National University of Defense Technology give model and algorithm of multiple satellites mission plan on ground and develops an integrated mission plan system on ground which is applied to the daily control and management of remote sensing satellites [5]. Yet currently this system is mainly employed on the ground and applied to non-agile satellites. TIAN Zhi-xin from Beijing institute of Spacecraft System Engineering put forward the algorithm of generating satellite mission based on directed graph model [6], which initiated the disintegration of instructions from mission level to execution level so that the complexity of satellite control was effectively reduced and improved the data injection efficiency of satellites. This method, however, is mainly aimed at non-agile satellites without taking the sophisticated imaging mission like active scan and corridor scan of agile satellites into consideration.

This paper analyzes the features of the autonomous mission management system of agile satellites first, and then demonstrates the four capabilities essential to the autonomous mission management system: mission planning, real-time high frequency calculation of the image parameter (the integral time), mission replanning and autonomous state monitoring throughout the procedure of imaging. At the same time, this paper studies the critical techniques which includes mission plan technique, emergency replan technique, image parameter real-time calculation technique, autonomous monitoring technique. The autonomous mission management system has been used on certain agile remote sensing satellite platform, which meets the requirements of multi-mode and multi-mission per revolution imaging for agile satellites. 


\section{The features of onboard autonomous mission management system}

Compared with the traditional satellites mission management system, the agile satellites autonomous mission management system has the following features:

1) More missions per revolution should be supported, requiring a stronger ability of onboard mission planning.

An agile satellite has a higher imaging efficiency and shorter mission switching, the number of the mission per revolution has a large increase compared with traditional satellite. The number of telecommand to upload will increase in exponential level if still use the traditional way of satellite control, can't meet the requirement of uploading all telecommand in a limited observe and control time window, so the onboard autonomous mission management system should have the ability of converting the telecommand from high mission level to low control level, to provide technology support for more imaging target.

2) The imaging mode becomes more complicated, requiring the satellite has the ability of real-time high frequency calculation of the camera parameter (integral time).

In addition to the imaging mode of passive scanning, an agile satellite also supports many complicated imaging mode like active scanning, scanning not along the $\operatorname{track}^{[7]}$, etc.(see Figure 1), the attitude changes flexible during the imaging procedure, the satellite can move on three axes and the angle velocity of the three axes can be nonzero. When the satellite images with nonzero angle rate, the severe change of slant distance not only brings the scale change in image, but also the change of image motion's angle velocity, resulting in the severe changes of integral time parameters during the imaging procedure. So the onboard autonomous mission management system should have the ability of real-time high frequency calculation of the integral time, so as to guarantee the imaging quality in agile mode.
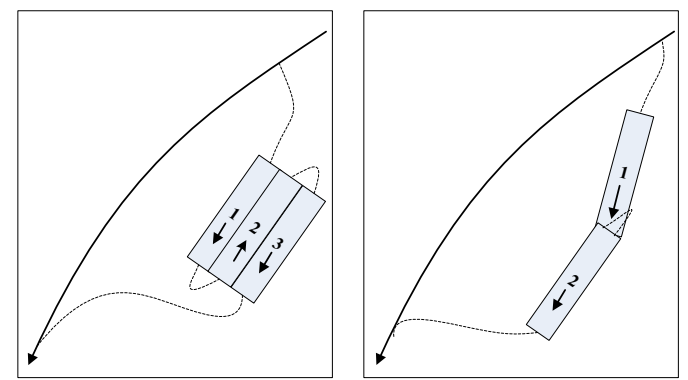

Figure 1. Complicated imaging mode of agile satellite.

3) The response speed of the emergency mission becomes faster, requiring the satellite has the ability of mission replanning.

The agile satellite has a stronger ability of attitude control, more flexible imaging, faster task switching, so the onboard autonomous mission management system should have the ability of mission replaning, providing rapid response services for users' emergency mission, to enhance the emergency support capability of satellite effectively.

4) The processing logic of the satellite becomes more complicated, requiring the satellite has a stronger ability of autonomous monitoring throughout the mission execution.

As agile satellites is required to implement more missions, to have more sophisticated imaging modes and to respond to emergencies more rapidly, there is greater probability of satellite abnormity throughout the mission execution, which in turn makes the capability of autonomous monitoring of the mission execution necessary for the satellites, so as to ensure the operation of satellite safe and reliable.

\section{The research of key technology}

As can be seen from the discussion about the features of autonomous mission management system, an agile satellite has a variety image mode, complicated data flow control, one of the key technology of the system is how to design a good system architecture. Secondly, the system should breakthrough three key technology including mission planning and replanning, real-time calculation of the integral time and autonomous monitoring throughout the mission execution, enabled the system has the four kind of ability discussed above.

\subsection{Layered architecture design of autonomous mission management system}

Since the Autonomous Mission Management System of agile satellites is characterized by diverse missions and difficult control of information flow, a series of proper system structures are demanded to facilitate the modular design of the system and effectively diminish the complexity of the execution of the system. As is displayed in Figure 2, this paper employs the idea of layered architecture to divide the system into application layer, planning layer, service layer and execution layer. On-board computer fulfills the planning, scheduling and execution of agile imaging in a layered progressive and gradually detailing way.

(1) Application Layer

This layer is composed of two modules, meta task receive module and meta task pre-process module. The meta task recerve module receives the demands from the ground, conducts batch management and submit the demands to meta task pre-process module on the basis of orbit time period. The module of pre-process sequences the to-be-planned meta task of current batch in time order, do preliminary check (e.g. the intervals of meta tasks, accumulated time length of imaging, etc. ) and form the logical sequence of meta tasks to be used by mission plan module.

\section{(2) Planning Layer}

This layer contains three modules, mission plan module, mission replan module and instruction generating module. Mission plan module receives meta 
tasks from the pre-process module of application layer, invokes the mission planning algorithm and outputs mission plan. The instruction generating module transform the mission plan into instructions that can be directly executed. The mission replan module deals with emergency mission from the ground, generate renewed plan and invokes the instruction generating module to produce instructions.

(3) Service Layer

This layer includes two modules, module of autonomic computing of parameter and module of mission execution monitoring. Module of autonomic computing of parameter provides parameters as integral time for mission plan and mission replan. Module of mission execution monitoring the running state of the satellites and rejects the instructions that cannot be executed.

\section{(4) Execution Layer}

This layer is formed of two modules, instruction verifying module and instruction execution module. Instruction verification makes judgment on the safety of the instructions sending safe instructions to the instruction execution module and rejecting unsafe ones. Instruction execution module activates the hardware of the satellite directly.

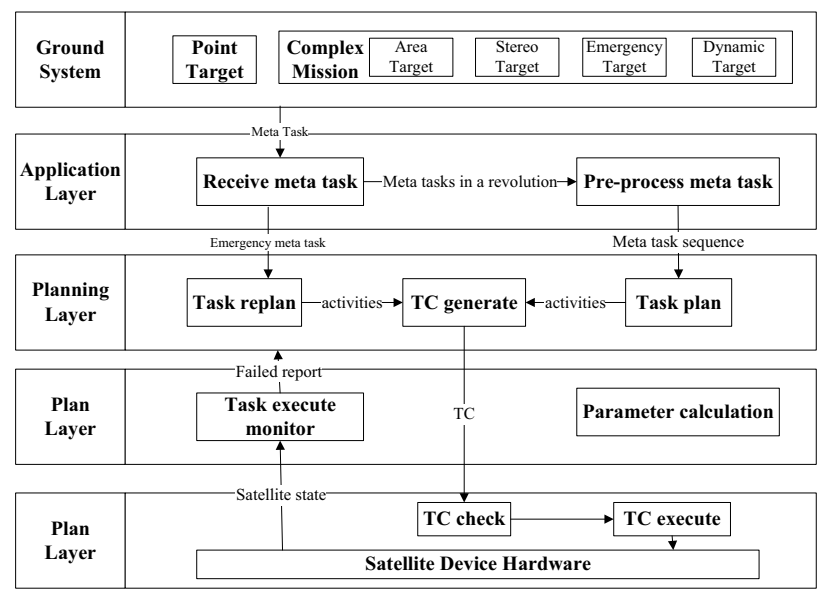

Figure 2. Architecture of autonomous mission management system.

\subsection{Techniques of mission plan and emergency replan}

To meet the imaging mission requirement, the agile satellite performs multiple different mode per revolution, the system needs the ability of rapidly mission planning. In order to reduce programming complexity and facilitate the implementation onboard, this paper adopts the strategy of planning missions next track before the track starts and form a preliminary plan. The planning process builds the constraint model of attitude control, power, storage first, planning algorithm calls constraint model to process the constraint checking of the missions contain. Instruction generation module rapidly translated the planning action sequence into the instruction can be directly executed according to the instruction template. If the system receives emergency meta task, it needs to insert the rapid emergency meta task into planned meta task queue in time order and analysis relations of conflict with the existing meta task time constraint. Deleting the existing tasks until meet the time constrains if there are conflicts and forming a feasible sequence of meta tasks. Then performing the mission planning process again (including constrains checking and action planning) and forming a task action sequence .The instruction generation module rapidly translated the planning action sequence into the instruction can be directly executed according to the instruction template at last. The whole procedure is shown in Figure 3.
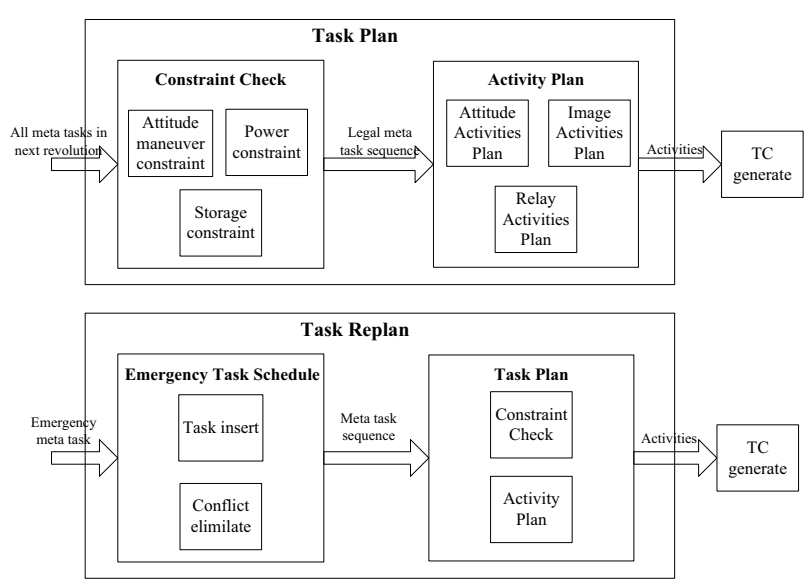

Figure 3. The procedure of mission planning and replanning.

\subsection{Techniques of onboard real-time calculation of the integral time}

Integral time is an import parameter for TDICCD camera in agile satellite. The accuracy of its value will affect image quality directly. The attitude of agile satellite changes quickly which lead the value of integral time wave largely, so traditional calculate mode cannot meet the requirement of agile satellite. We modify the velocity and height mode on paper [8], and use the new mode to calculate the integral time in agile satellite.

\subsubsection{The modification of shoot point velocity mode}

The attitude angle rate was nonzero when image in agile mode, so we should consider attitude angel rate when calculate vector of satellite coordinate angel rate. The calculate formula is:

$$
w_{\mathrm{b}}=w_{\mathrm{n}}+w_{\mathrm{s}}
$$

$w_{\mathrm{b}}$ is the vector of satellite coordinate angle rate, $w_{\mathrm{n}}$ is the vector of orbit angle rate, $w_{\mathrm{s}}$ is the vector of satellite attitude angle rate

So the shoot point velocity mode of agile satellite has difference compared to the traditional calculate mode, the calculate formula is:

$$
v=w_{\mathrm{e}} \times R-\left(w_{\mathrm{n}} \times r+w_{\mathrm{b}} \times H+v_{\mathrm{r}}\right)
$$

$w_{\mathrm{e}}$ is the vector of earth rotation angle rate, $\mathrm{R}$ is the vector from earth center point to shoot point, $r$ is the 
vector from earth center point to satellite, $H$ is the vector from satellite to shoot point, $v_{\mathrm{r}}$ is the vector of satellite speed along to the earth center point.

\subsubsection{The modification of height mode}

Height calculate mode is a simple and low-accuracy mode, it doesn't consider the ground height, we should use the digital ground height diagram to modify the height between satellite and shoot point for more accurate integral time value. Figure 4 shows the new calculate mode.

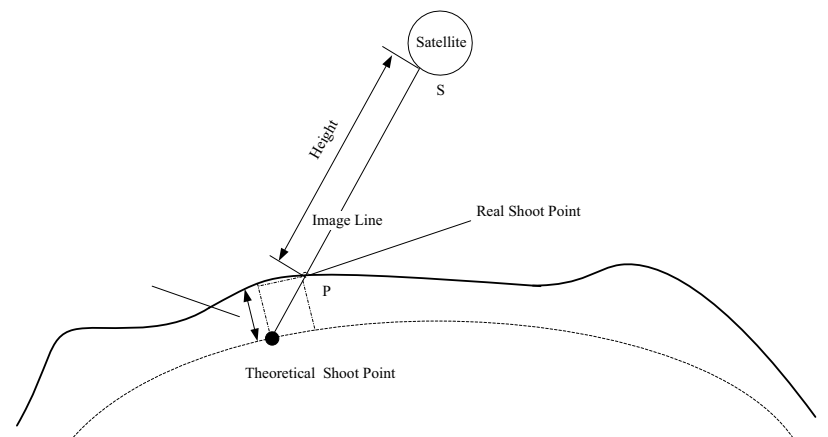

Figure 4. The new height calculate mode.

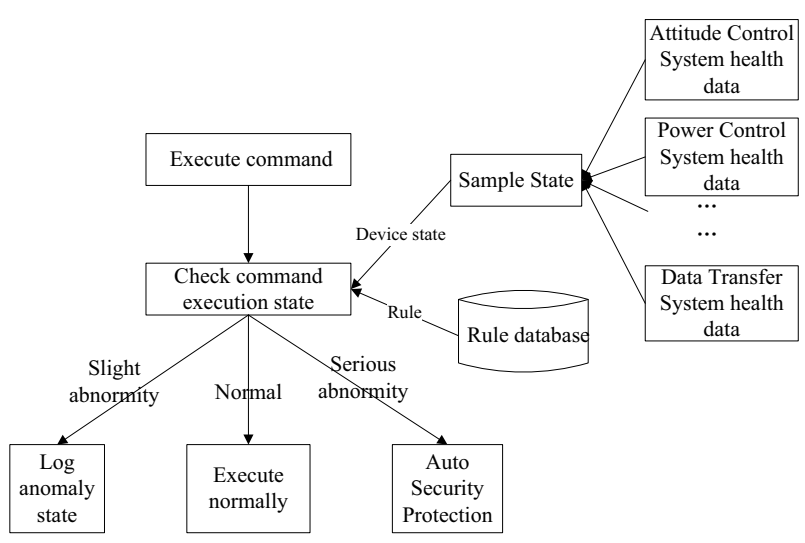

Figure 5. The procedure of autonomous monitoring throughout the mission execution.

\subsection{Techniques of autonomous monitoring of mission execution}

As agile satellites is required to implement more missions, to have more sophisticated imaging modes and to respond to emergencies more rapidly, there is greater probability of satellite abnormity throughout the mission execution, which in turn makes the capability of autonomous monitoring of the mission execution necessary for the satellites. The fundamental part of the mission execution monitoring is to guarantee safe execution of each instruction of each subsystem during the mission, thus real-time checking of the results of each instruction is necessary. State sampling module collects real-time state of each subsystem, with the integration of the judgment rule database in the computer memory of the satellites, to make judgments on the normality of the instruction execution. Slight abnormity is logged and regularly sent back to the ground by event reports so that the designer can analyze the cause of the abnormity. If there is serious abnormity, autonomous protection mode will be switched on and the satellite loading will be safely shut down, in which way the satellite will be running safely at the minimum function level. If the execution is normal, next instruction will be executed. The whole procedure is illustrated in Figure 5.

\section{Applications}

Key technology based design of autonomous mission management system has been applied in an agile platform of remote sensing satellite and its data flow diagram is showed in Figure 6.

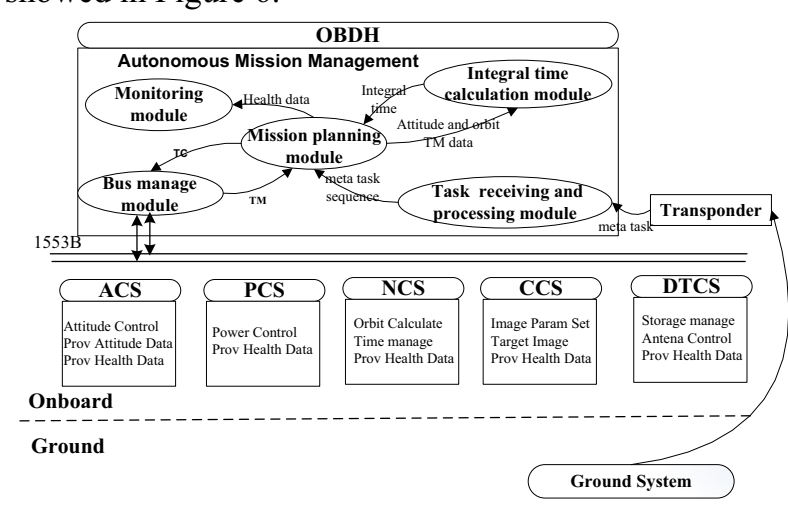

Figure 6. Data flow diagram of autonomous mission management system in an agile platform.

Onboard transponder receives meta task from ground and sends them to OBDH subsystem. OBDH subsystem using these meta task data and each subsystem TM data including attitude data, orbital data and subsystem health data etc., from $1553 \mathrm{~B}$ bus to complete mission management functions such as the mission planning, integral time calculation, mission execution monitoring. Current system operation condition conforms to expectation design and the system can meet the multimode multi-mission per revolution imaging needs of an agile satellite. Compared with traditional remote sensing satellite, the advantages of using onboard autonomous mission management functions are shown in Table 1.

Table 1. Comparison of applied and note applied the onboard autonomous mission management.

\begin{tabular}{|c|c|c|}
\hline $\begin{array}{c}\text { The number of missions per } \\
\text { revolution }\end{array}$ & Applied & Not-applied \\
\hline Task constrained check ability & Yes & Now \\
\hline $\begin{array}{c}\text { Single Mission injection data } \\
\text { volume/Byte }\end{array}$ & 40 & 220 \\
\hline $\begin{array}{c}\text { Rapid response ability to } \\
\text { Emergency Mission }\end{array}$ & Yes & No \\
\hline $\begin{array}{c}\text { Integral time real-time frequent } \\
\text { calculation ability }\end{array}$ & Yes & No \\
\hline $\begin{array}{c}\text { Mission autonomous monitoring } \\
\text { ability }\end{array}$ & Yes & No \\
\hline
\end{tabular}




\section{Conclusions}

High autonomy is a significant field in spacecraft development. Comparing with TM\&TC based traditional mission operation and management mode, onboard autonomous mission management technology can sharply improve the satellite whole application efficiency and reduce the cost of operation and management on the ground. This paper proposes an autonomous mission management system onboard based on the key technologies researched, the system has been used in certain agile platform and will meet the requirement of new earth observing satellites in future.

\section{References}

1. S.Chie, G.Rabideau. ASPEN-auto-mated planning and scheduling for space mission operations $[\mathrm{C}] / / 6^{\text {th }}$ International Symposium on Space Missions Operations and Ground Data System. France, Toulouse, 2000.

2. Cichy B, Chien S. Validating the EO-1 autonomous science agent[C]//International Workshop on Planning and Scheduling for Space. Germany, Darmstadt, 2004.
3. Bamsley MJ, Settle JJ, Cuter MA, te al.The PROBA/ CHRIS mission: a low-Cost smallsat for hyperspectral multiangle observations of the earth surface and atomosphere[J]. IEEE Transactions on Geoscience and Remote Sensing, 2004, 42(7): 15121520 .

4. Biachessi N.Planning and Scheduling problems for earth observing satellites: models and algorithms[D]. Milano: Universita degli Studi di Milano,2006.

5. HE Ren-jie, GAO Bao-cun, LI Ju-fang. Models, algorithms and applications to the mission planning system of imaging satellite[J] Systems engineering Theroy\&Practice, 2011,31(3): 412-413(in Chinese)

6. TIAN Zhixin, Cui Xiaoting. Remote Sensing Satellite Autonomous Command Sequences Generation Based on Digraph-model[J]. Spacecraft Engineering, 2014, 23(6):55-57(in Chinese)

7. ZHANG XinWei, DAI Jun. Research on Working Mode of Remote Sensing Satellite with Agile Attitude Contro 1[J]. Spacecraft Engineering, 2011, 20(4) :34-37(in Chinese).

8. CHEN Shaolong Computation Model of Veer Angle and Velocity - Height Ratio for Space Camera in Roll Attitude [J]. Spacecraft Engineering, 2010, 19(1):38-39 (in Chinese) 